\title{
'Candidatus Liberibacter' Pathosystems at the Forefront of Agricultural and Biological Research Challenges
}

\author{
Elizabeth A. Pierson, ${ }^{1, \dagger}$ Jaime Cubero, ${ }^{2, \dagger}$ Caroline Roper, ${ }^{3, \dagger}$ Judith K. Brown, ${ }^{4, \dagger}$ Clive H. Bock, ${ }^{5, \dagger}$ and Nian Wang ${ }^{6, \dagger}$ \\ ${ }^{1}$ Department of Horticultural Sciences, Texas A\&M University, College Station, TX 77843-2133 \\ ${ }^{2}$ Departamento de Protección Vegetal, Laboratorio Bacteriología, Centro Instituto Nacional de Investigación y Tecnología Agraria y Alimentaria \\ (INIA/CSIC), Madrid 28040, Spain \\ ${ }^{3}$ Department of Microbiology and Plant Pathology, University of California-Riverside, Riverside, CA 92521 \\ ${ }^{4}$ School of Plant Sciences, The University of Arizona, Tucson, AZ 85721 \\ ${ }^{5}$ United States Department of Agriculture, Agriculture Research Service, Southeastern Fruit and Tree Nut Research Station, Byron, GA \\ 31008 \\ ${ }^{6}$ Citrus Research and Education Center, Department of Microbiology and Cell Science, Institute of Food and Agricultural Sciences, \\ University of Florida, Lake Alfred, FL 33850 \\ Accepted for publication 2 December 2021
}

'Candidatus Liberibacter' species are Gram-negative, phloemcolonizing, psyllid-transmitted fastidious bacteria, classified in the Rhizobiaceae (class Alphaproteobacteria) (Bové 2006). Thus far, seven Liberibacter species have been identified worldwide (Hansen et al. 2008; Jagoueix et al. 1994, 1997; Leonard et al. 2012; Liefting et al. 2008; Morris et al. 2017; Raddadi et al. 2011; Texeira et al. 2005; Thompson et al. 2013). Only one Liberibacter species, L. crescens, has been cultured axenically in artificial media, which renders Candidatus status for other Liberibacter species. $L$. crescens, was isolated from mountain papaya in Puerto Rico but it does not appear to be pathogenic to papaya and the bacterial-host interaction has not been investigated in detail. In contrast, modified Koch's postulates have been fulfilled for several ' $\mathrm{Ca}$. Liberibacter' species, including ' $C a$. L. asiaticus' (CLas), corroborating the status of ' $C a$. Liberibacter' species as causal agents of plant diseases. CLas, ' $\mathrm{Ca}$. L. africanus' (CLaf), and ' $\mathrm{Ca}$. L. americanus' (CLam) are the causal agents of citrus huanglongbing (HLB) or citrus greening disease, which is the most devastating citrus disease worldwide (Wang 2019). CLas and CLam are transmitted by the Asian citrus psyllid Diaphorina citri Kuwayama, and CLaf by the African citrus psyllid Trioza erytreae Del Guercio (Capoor et al. 1967; McClean and Oberholzer 1965). Within the ' $\mathrm{Ca}$. L. solanacearum' (CLso) (syn. 'Ca. L. psyllaurous') species, 12 haplotypes have been described to date (Mora et al. 2021). Haplotypes CLso A and $\mathrm{B}$ infect solanaceous crops of which potato and tomato are the most economically important. Potato plants affected by zebra chip disease exhibit tuber discoloration, whereas vein-greening disease of tomato affects fruit production. Both haplotypes are transmitted by the potato-tomato psyllid Bactericera cockerelli Sulc. and are endemic to North America. CLso A is also established in New Zealand following an accidental introduction. Haplotypes CLso C, D, and $\mathrm{E}$ occur in Europe and/or the Mediterranean Basin and are transmitted by the carrot psyllid T. apicalis Förster or the leekonion psyllid B. tremblayi Wagner (Munyaneza et al. 2010; Nelson et al. 2011) (and perhaps other psyllids). The haplotypes CLso C, $\mathrm{D}$, and $\mathrm{E}$ have host plants in the Apiaceae, including celery, carrot, parsley, and parsnip. In addition, haplotypes CLso $\mathrm{C}$ and $\mathrm{E}$ have been occasionally found infecting potato plants in Finland and

${ }^{\dagger}$ Corresponding authors: elizabeth.pierson@ag.tamu.edu, cubero@inia.es, mcroper@ucr.edu,jbrown@ag.arizona.edu, clive.bock@usda.gov, and nianwang@ufl.edu

The author(s) declare no conflict of interest.

(C) 2022 The American Phytopathological Society
Spain. Haplotypes CLso F and G have been detected in the United States in a single potato tuber and from herbarium specimens of Solanum umbelliferum Eschsch., respectively. More recently, haplotype CLso $\mathrm{H}$ has been identified infecting species of Polygonaceae and Convolvulaceae, whereas haplotype CLso U was identified from Urtica dioica L. in Finland. Haplotypes Cra1 and Cra2 were found infecting psyllids belonging to the Aphalaridae, but they have not been associated with a plant disease. Two additional ' $\mathrm{Ca}$. Liberibacter' species discovered recently are ' $\mathrm{Ca}$. L. europaeus' and ' $\mathrm{Ca}$. L. brunswickensis'. The former is indigenous to Western Europe where it associates with Scotch broom plants and pear trees and is transmitted by the pear/broom psyllid Cacopsylla pyri L., and the latter, ' $\mathrm{Ca}$. L. brunswickensis', is associated with the Australian eggplant psyllid Acizzia solanicola Kent \& Taylor (Morris et al. 2017). Whether ' $C a$. L. europaeus' and ' $C a$. L. brunswickensis' are plant pathogens is not known.

The fastidious ' $\mathrm{Ca}$. Liberibacter' pathogens and their psyllid vectors are associated with economically important plant diseases and are apparently geographically expanding worldwide. Consequently, ' $\mathrm{Ca}$. Liberibacter' pathosystems have attracted tremendous attention from growers and the respective agricultural industries, the public, the scientific community, and government agencies. Resources have been directed at understanding the nature of the diseases caused by ' $\mathrm{Ca}$. Liberibacter' species to accelerate the development of short- and long-term management approaches to combat losses due to damage caused by psyllid vector feeding and ' $\mathrm{Ca}$. Liberibacter' infection. Presently, management relies primarily on routine insecticide applications to reduce psyllid vector population size to lower transmission frequency. Insecticides are expensive, variably successful, and not ecologically sound nor economically sustainable. Further, ' $\mathrm{C} a$. Liberibacter' pathosystems present many research challenges. First, because ' $\mathrm{Ca}$. Liberibacter' species have not been cultured on artificial media, traditional molecular and genetic studies have been generally limited to indirect analyses. Consequently, progress has been slower than desirable in understanding ' $\mathrm{Ca}$. Liberibacter' genetics in general and with respect to elucidating molecular mechanisms for pathogenesis in the plant and psyllid hosts. Second, because ' $\mathrm{Ca}$. Liberibacter' species reside only in specialized living environments in the host plant (i.e., phloem) or vector (specific psyllid cells, tissues, and organs) that are difficult to manipulate, critical studies of host-microbe interactions have required the development of new tools for visualization, manipulation, and molecular characterization.

Despite the difficulties surrounding the study of ' $\mathrm{Ca}$. Liberibacter' pathosystems, these unusual microbes that infect both plant and insect hosts present unique opportunities to explore intriguing questions. How common are ' $\mathrm{Ca}$. Liberibacter' species as endophytes 
among eudicot plants? In which hosts do they function as plant pathogens and why? How did the ancestors of ' $\mathrm{Ca}$. Liberibacter' species establish associations with plants and what mechanisms enable them to specialize in the host phloem? How did ' $\mathrm{Ca}$. Liberibacter' species establish associations with psyllids and what mechanisms enable them to exploit the different life stages and facilitate psyllid-specific transmission in a circulative, propagative manner? How do their host associations influence in planta disease incidence, symptom development, and severity? Can they be cultured in artificial media? Until now, studies of plant-bacterial interactions have focused primarily on extracellular bacterial pathogens. Research on ' $\mathrm{Ca}$. Liberibacter' pathosystems also offers a timely opportunity to probe phloem biology, including phloem transport. The ' $\mathrm{Ca}$. Liberibacter' pathosystems provide unique opportunities to study the interactions that have given rise to ' $\mathrm{Ca}$. Liberibacter'-psyllid-plant coevolution. Ultimately, a better understanding of the "dual host" pathosystem is essential to enable the design and implementation of effective, economical, and sustainable solutions for disease management.

The recent emergence of previously unknown ' $\mathrm{Ca}$. Liberibacter'psyllid-plant complexes that cause unprecedented yield- and qualitylimiting diseases of diverse crop species has elevated this pathosystem to the forefront of investigation among plant pathogens, and specifically in the plant pathogen-insect vector arena. Thus, exciting new insights are eagerly anticipated from research into biological, genetic, genomic, and evolutionary aspects of ' $\mathrm{Ca}$. Liberibacter'-plant-psyllid interactions. Given the economic importance, broad impact on plant pathology and biology, and potential importance of better management based on new knowledge of ' $\mathrm{Ca}$. Liberibacter'-plant-insect interactions, Phytopathology was compelled to select ' $\mathrm{Ca}$. Liberibacter' pathosystems as the topic for the January 2022 Focus Issue.

We are delighted to present several reviews and original research articles by leading scientists covering a wide range of topics related to ' $C a$. Liberibacter' pathosystems. We begin with four informative reviews summarizing essential current knowledge of HLB pathosystems or disease management.

The review by Pandey et al. (2022) reports current knowledge of molecular mechanisms of ' $\mathrm{Ca}$. Liberibacter' pathogenesis. The review of current literature is refreshingly organized around simple questions that align with key points of control in host-pathogen interactions. They focus first on the question of whether the bacteria are motile within insect and plant hosts, the roles of flagella and type IV pili, and the biosynthesis pathways involved. They also address how ' $\mathrm{Ca}$. Liberibacter' manipulates host global gene expression patterns, metabolism, and defense responses via their extensive review of plant genomics and proteomics studies. Pandey et al. (2022) further address how the pathogen adapts to the plant host niche including the modulation of its own genome. Lastly, they revisit the idea that bacterial movement in the host is important for pathogenicity and provide insights into how pathogen movement influences disease and symptom development in host plants.

Recognizing the importance of the dual lifestyle of the bacteria within its plant and insect hosts, Killiny (2022a) provides a review of the molecular and biochemical aspects of the interaction cycle between CLas and the Asian citrus psyllid D. citri. Much of the review focuses on the effects of CLas on the metabolic profile of $D$. citri. Specific emphasis is placed on psyllid genes/pathways involved in energetics, metabolic pathways, defense response, detoxification during infection, and modulation of the immune system of $D$ citri. The author describes effects of CLas infection on D. citri endosymbiont microbiota as well as effects of the bacteria on D. citri fitness, host preference, and behavior, leading to enhanced vector dispersal and bacterial transmission. Although some authors have considered CLas $-D$. citri interactions benign, evidence is emerging that ' $C a$. Liberibacter' infection results in a shortened lifespan but higher fecundity, and alters feeding behavior in favor of CLas transmission, in short, a mutualistic relationship with tradeoffs experienced by both partners. da Graça et al. (2022) offer an excellent historical perspective on the CLaf-citrus pathosystem in Africa, including information on CLaf and greening disease, CLaf distribution, CLaf subspecies causing noncitrus infection, and CLaf detection and HLB management in Africa. Throughout this review the authors draw comparisons between CLaf and CLas, which provides interesting insight into differences in the biology and pathogenicity of the two HLB pathogens.

Alquézar et al. (2022) provide a broad review of cultural approaches used to control HLB. The authors analyze the pros and cons of control approaches including area wide management programs to control the psyllid vector, citrus under protective screen (CUPS) production systems and biological control through release of the Asian citrus psyllid parasitoid Tamarixia radiata Waterston. Importantly, the authors compare HLB control in different scenarios based on psyllid infestation and HLB status.

Contributing further to our understanding of ' $\mathrm{Ca}$. Liberibacter' biology are two original research articles. The paper by De Francesco et al. (2022) describes use of an elegant bacterial cell enrichment procedure for transcriptome profiling of CLas in citrus and psyllids. The method circumvents common problems related to low coverage of the CLas genome in dual-transcriptome analyses, providing expression profiles of approximately $84 \%$ of the CLas genes and facilitating comparative perspective of expression patterns relative to host adaptation. Original research by Armstrong et al. (2022) identified a unique genetic element ( $\sim 8.3 \mathrm{~kb}$ DNA) that is variably present within the CLas genome and report on its prevalence in CLas populations in psyllids and citrus. Although samples from psyllids and citrus often ( $\sim 90 \%$ of the time) contained mixed populations of wild type (having the genetic element) and mutants (lacking the genetic element), populations of wild type alone were detected at a low rate in psyllids but not in citrus, whereas populations of the mutant alone sometimes occurred in citrus but not psyllids. The results indicate that this unique genetic element may be a useful marker for population studies. The authors conclude with the intriguing hypothesis that phase variation, or "reversible genetic alterations" at specific loci within genomes may be a possible mechanism for host adaptation and virulence.

Two studies examined the effects of CLas infection on host metabolism. Padhi et al. (2022) provide a longitudinal overview of the metabolic effects of CLas infection of 'Lisbon' lemon trees via psyllid transmission using proton-nuclear magnetic resonance spectroscopy. Significant differences were observed between the leaf metabolome of control versus infected trees during the early phases of CLas infection including alterations in glucose and quinic acid concentrations. Vital to the future of genetic-based research on ' $\mathrm{Ca}$. Liberibacter' pathosystems is the ability to culture ' $\mathrm{Ca}$. Liberibacter' species. To better understand the nutritional requirements of CLas, research by Killiny (2022b) focused on differences between CLas-infected and noninfected plants. The chemical composition of phloem sap from healthy and CLas-infected plants was compared using GC-MS-based methods in two different plant hosts, citrus and periwinkle. Compared with noninfected plants, the concentrations of certain organic acids, amino acids, and tricarboxylic acid (TCA) cycle metabolites were lower in the CLas infected phloem sap from both species. One hypothesis that remains untested is that the changes may reflect utilization of host resources by CLas to fulfill its nutritional needs, and if so, the results may hint at the nutritional requirements for axenic growth of CLas in artificial media. Further innovative work by Merfa et al. (2022a) focused on methods to culture CLas. They report methods to optimize CLas growth in grapefruit juice amended with a variety of compounds and incubation over a range of conditions. An unexpected finding was that CLas population growth was inversely proportional to the initial inoculum concentration, suggesting a cell density-dependent mechanism regulating growth. The authors report on successes in enhancing growth rates and population levels, although the cultures were viable only transiently. The authors also report ongoing progress to develop a system to grow CLas within periwinkle calli in the laboratory. 
Three studies investigated the dynamic association of ' $\mathrm{Ca}$. Liberibacter' with the plant host or psyllid vector. Research by Gasparoto et al. (2022) examined the possible reasons for the prevalence of CLas compared with CLam observed in citrus orchards of São Paulo, Brazil, where both species and the insect vector D. citri are present. The authors discuss their findings in the context of what is known about how biological differences between the two species and their association with citrus influence psyllid-mediated acquisition and transmission. Ramsey et al. (2022) investigated the effect of host switching by D. citri between Citrus medica and Murraya paniculate, a citrus relative previously shown to be more resistant to CLas compared with all tested Citrus genotypes. Host switching altered the acquisition and transmission of CLas as well as its effect on $D$. citri protein expression. The authors have suggested that the information learned could shed light on vector competence and HLB disease management. Additionally, Wamonje et al. (2022) provide the first evidence that CLso is associated with the cottony ash psyllid Psyllopsis discrepans Flor colonizing ash trees. The recent discovery warrants further investigation, given recent episodes of dieback of ash in Saskatchewan associated with psyllid feeding and symptoms that are consistent with infection by other 'Ca. Liberibacter' species.

Returning to the importance of understanding pathogen-vector interactions, the study by Lin et al. (2022) focuses on the intracellular cycle of CLas in midgut cells of $D$. citri. Through elegant immunolocalization and microscopy studies, the authors demonstrate that CLas employs endo/exocytosis-like mechanisms that mediate bacterial invasion and egress in the midgut. In addition, they observed differential expression of autophagy-related genes in CLas-infected psyllids suggesting that CLas subverts host autophagy in its psyllid vector to promote infection. These results indicate that CLas intimately interacts with its psyllid host and undergoes a multistage intracellular cycle through endocytic, secretory, autophagic, and exocytic pathways.

The study by Merfa et al. (2022b) focuses on the potential role of the bacterial outer membrane protein in OmpA in pathogenvector interactions and investigates the possibility of using OmpAderived peptides to disrupt CLas acquisition by D. citri. Several of the OmpA-derived peptides significantly reduced the population of CLas acquired by $D$. citri, and at a concentration of $50 \mu \mathrm{M}$ in the artificial diet completely disrupted of CLas acquisition by adult psyllids. Spray application of $50 \mu \mathrm{M}$ of Pep6, to orange plants, also significantly reduced CLas acquisition by adult psyllids. The study demonstrates the utility of a biotechnological approach targeting pathogen-vector molecular interactions, and the potential for use of OmpA-derived peptides to control CLas.

The study by Igwe et al. (2022) introduces a streamlined excised leaf CLas acquisition assay that enables rapid, accurate measurements of CLas acquisition by $D$. citri. Compared with most of the ongoing work focusing on acquisition by adults, a novel and exciting component of the work is the focus on healthy fourth and fifth instar nymphs. The authors suggest this useful method could be used to facilitate studies of psyllid and plant factors contributing to CLas acquisition, as well as to test antimicrobial compounds that interfere with CLas acquisition.

Two articles, including one long-term study, provided new epidemiological knowledge of factors that contribute to disease progress and spatial distribution, providing fresh insight for disease management. Alves et al. (2022) collected HLB presence/absence data in citrus orchards in Minas Gerais, Brazil, over a 13-year timeframe, and compared two hierarchical Bayesian modeling approaches to link climatic factors to the spatial distribution of HLB prevalence. Both modeling approaches showed an inverse relationship between regional prevalence of HLB and mean temperature during the dry season. Other factors, including annual wind speed, and annual and rainy season rainfall, and neighboring area HLB status, contributed to HLB prevalence, furthering the understanding of environmental factors associated with distribution and spread of the disease. The new information could assist policymakers in defining regions at risk for HLB outbreaks to guide monitoring strategies to mitigate further spread of HLB in high-risk areas. In addition, Keshet-Sitton et al. (2022) provide insights into disease development in carrot associated with CLso haplotype D in Israel (CLso D). The effects of plant age, growth temperature, and vector load on shoot proliferation symptoms were studied. They discuss their findings in the context of environmental effects and time required for CLso D populations to reach an infection threshold.

Given the importance of effective insect vector control in disease management, we were pleased to include the study by Menger et al. (2022) who used a unique fluorescence method to evaluate insecticide distribution in citrus groves. The upper surface of the leaves received significantly more insecticide compared with the lower surface of the leaves, and application by ground-based tractor sprayer was more effective than aerial application for ensuring adequate insecticide coverage. Results from the study should provide useful information for the development of strategies to optimize spray application for pest management to effectively target psyllids.

Overall, the collection of articles in this focus issue showcases the enormous research efforts made by the scientific community on ' $\mathrm{Ca}$. Liberibacter' pathosystems, giving rise to major advances and achievements in a short time often through multidisciplinary approaches applied to the bacterium, psyllid vector, and plant host. We hope this focus issue will be a useful and valuable resource of information and inspiration for future research in ' $\mathrm{Ca}$. Liberibacter'disease management, with the realization that effective control relies on new discoveries and a greater understanding of multiple facets of ' $C a$. Liberibacter' pathosystems that remain poorly understood. We would like to thank the contributing authors for a series of exceptional articles and the anonymous reviewers who provided valuable feedback.

\section{LITERATURE CITED}

Alquézar, B., Carmona, L., Bennici, S., Miranda, M. P., Bassanezi, R., and Peña, L. 2022. Cultural management of huanglongbing: Current status and ongoing research. Phytopathology 112:11-25.

Alves, K. S., Rothmann, L., and Del Ponte, E. 2022. Linking climate variables to large-scale spatial pattern and risk of citrus huanglongbing: A hierarchical Bayesian modeling approach. Phytopathology 112:189-196.

Armstrong, C. M., Zhou, L., Luo, W., Batuman, O., Alabi, O. J., and Duan, Y. 2022. Identification of a chromosomal deletion mutation and dynamics of two major populations of 'Candidatus Liberibacter africanus' in its hosts. Phytopathology 112:81-88.

Bové, J. M. 2006. Huanglongbing: A destructive, newly-emerging, centuryold disease of citrus. J. Plant Pathol. 88:7-37.

Capoor, S. P., Rao, D. G., and Viswanath, S. M. 1967. Diaphorina citri Kuway., a vector of the greening disease of citrus in India. Indian J. Agric. Sci. 37:1382.

da Graça, J. V., Cook, G., Ajene, I. J., Grout, T. G., Pietersen, G. Roberts, R., Bester, R., Pretorius, M. C., and Maree, H. J. 2022. A review of the 'Candidatus Liberibacter africanus' citrus pathosystem in Africa. Phytopathology 112:44-54.

De Francesco, A., Lovelace, A. H., Shaw, D., Qiu, M., Wang, Y., Gurung, F., Ancona, V., Wang, C., Levy, A., Jiang, T., and Ma, W. 2022. Transcriptome profiling of 'Candidatus Liberibacter asiaticus' in citrus and psyllids. Phytopathology 112:116-130.

Gasparoto, M. C. G., Primiano, I. V., Bassanezi, R. B., Lourenço, S. A., Montesino, L. H., Wulff, N. A., Martins, E. C., Bergamin Filho, A. B., and Amorim, L. 2022. Prevalent transmission of 'Candidatus Liberibacter asiaticus' over ' $\mathrm{Ca}$. Liberibacter americanus' in a long-term controlled environment. Phytopathology 112:180-188.

Hansen, A., Trumble, J., Stouthamer, R., and Paine, T. 2008. A new huanglongbing species, "Candidatus Liberibacter psyllaurous," found to infect tomato and potato, is vectored by the psyllid Bactericera cockerelli (Sulc). Appl. Environ. Microbiol. 74:5862-5865.

Igwe, D. O., Higgins, S. A., and Heck, M. 2022. An excised leaf assay to measure acquisition of 'Candidatus Liberibacter asiaticus' by psyllids associated with citrus huanglongbing disease. Phytopathology 112: 69-75.

Jagoueix, S., Bove, J. M., and Garnier, M. 1994. The phloem-limited bacterium of greening disease of citrus is a member of the alpha subdivision of the Proteobacteria. Int. J. Syst. Bacteriol. 44:379-386. 
Jagoueix, S., Bove, J. M., and Garnier, M. 1997. Comparison of the 16S/23S ribosomal intergenic regions of "Candidatus Liberobacter asiaticum" and "Candidatus Liberobacter africanum", the two species associated with citrus huanglongbing (greening) disease. Int. J. Syst. Bacteriol. 47:224-227.

Keshet-Sitton, A., Piasetzky, A., Shoshana, N., Dror, O., and Bahar, O. 2022. Effect of plant age, temperature, and vector load on 'Candidatus Liberibacter solanacearum' in planta titer and shoot proliferation symptoms in carrot. Phytopathology 112:154-162.

Killiny, N. 2022b. Generous hosts: 'Candidatus Liberibacter asiaticus' growth in Madagascar periwinkle (Catharanthus roseus) highlights its nutritional needs. Phytopathology 112:89-100.

Killiny, N. 2022a. Made for each other: Vector-pathogen interfaces in the huanglongbing pathosystem. Phytopathology 112:26-43.

Leonard, M. T., Fagen, J. R., Davis-Richardson, A. G., Davis, M. J., and Triplett, E. W. 2012. Complete genome sequence of Liberibacter crescens BT-1. Stand. Genomic Sci. 7:271-283.

Liefting, L., Perez-Egusquiza, Z., Clover, G., and Anderson, J. 2008. A new 'Candidatus Liberibacter' species in Solanum tuberosum in New Zealand. Plant Dis. 92:1474.

Lin, C.-Y., Achor, D., and Levy, A. 2022. Intracellular life cycle of 'Candidatus Liberibacter asiaticus' inside psyllid gut cells. Phytopathology 112:145-153

McClean, A. P. D., and Oberholzer, P. C. J. 1965. Citrus psylla, a vector of the greening disease of sweet orange-research note. South African J. Agric. Sci. 8:297-298.

Menger, R. F., Rehberg, R. A., Trivedi, P., Henry, C. S., and Borch, T. 2022. High spatial resolution fluorescence imagery for optimized pest management within a huanglongbing-infected citrus grove. Phytopathology 112: 173-179.

Merfa, M. V., Fischer, E. R., de Sousa e Silva, M., Francisco, C. S., ColettaFilho, H. D., and de Souza, A. A. 2022b. Probing the application of OmpA-derived peptides to disrupt the acquisition of 'Candidatus Liberibacter asiaticus' by Diaphorina citri. Phytopathology 112:163-172.

Merfa, M. V., Naranjo, E., Shantharaj, D., and De La Fuente, L. 2022a. Growth of 'Candidatus Liberibacter asiaticus' in commercial grapefruit juice-based media formulations reveals common cell density-dependent transient behaviors. Phytopathology 112:131-144.

Mora, V., Ramasamy, M., Damaj, M. B., Irigoyen, S., Ancona, V., Ibanez, F., Avila, C. A., and Mandadi, K. K. 2021. Potato zebra chip: An overview of the disease, control strategies, and prospects. Front. Microbiol. 12:700663.
Morris, J., Shiller, J., Mann, R., Smith, G., Yen, A., and Rodoni, B. 2017. Novel 'Candidatus Liberibacter' species identified in the Australian eggplant psyllid, Acizzia solanicola. Microbiol. Biotechnol. 10:833-844.

Munyaneza, J. E., Fisher, T. W., Sengoda, V. G., Garczynski, S. F., Nissinen, A., and Lemmetty, A. 2010. Association of "Candidatus Liberibacter solanacearum" with the psyllid, Trioza apicalis (Hemiptera: Triozidae) in Europe. J. Econ. Entomol. 103:1060-1070.

Nelson, W. R., Fisher, T. W., and Munyaneza, J. E. 2011. Haplotypes of 'Candidatus Liberibacter solanacearum' suggest long-standing separation. Eur. J. Plant Pathol. 130:5-12.

Padhi, E. M. T., Araujo, K. J., Mitrovic, E., Polek, M., Godfrey, K. E., and Slupsky, C. M. 2022. The impact of Diaphorina citri-vectored 'Candidatus Liberibacter asiaticus' on citrus metabolism. Phytopathology 112:197-204.

Pandey, S. S., Hendrich, C., Andrade, M. O., and Wang, N. 2022. Candidatus Liberibacter: From movement, host responses, to symptom development of citrus huanglongbing. Phytopathology 112:55-68.

Raddadi, N., Gonella, E., Camerota, C., Pizzinat, A., Tedeschi, R., Crotti, E., Mandrioli, M., Bianco, P. A., Daffonchio, D., and Alma, A. 2011. 'Candidatus Liberibacter europaeus' sp. nov. that is associated with and transmitted by the psyllid Cacopsylla pyri apparently behaves as an endophyte rather than a pathogen. Environ. Microbiol. 13:414-426.

Ramsey, J. S., Ammar, E.-D., Mahoney, J. E., Rivera, K., Johnson, R., Igwe, D. O., Thannhauser, T. W., MacCoss, M. J., Hall, D. G., and Heck, M. 2022. Host plant adaptation drives changes in Diaphorina citri proteome regulation, proteoform expression, and transmission of 'Candidatus Liberibacter asiaticus', the citrus greening pathogen. Phytopathology 112:101-115.

Texeira, D. C., Ayres, J., Kitajima, E. W., Danet, L., Jagoueix-Eveillard, S., Saillard, C., and Bové, J. M. 2005. First report of a huanglongbing-like disease of citrus in Sao Paulo State, Brazil and association of a new Liberibacter species, "Candidatus Liberibacter americanus", with the disease. Plant Dis. 89:107.

Thompson, S., Fletcher, J., Ziebell, H., Beard, S., Panda, P., Jorgensen, N., Fowler, S., Liefting, L., Berry, N., and Pitman, A. 2013. First report of 'Candidatus Liberibacter europaeus' associated with psyllid infested Scotch broom. New Dis. Rep. 27:6.

Wamonje, F. O., Zhou, N., Bamrah, R., Wist, T., and Prager, S. M. 2022. Detection and identification of a 'Candidatus Liberibacter solanacearum' species from ash tree infesting psyllids. Phytopathology 112:76-80.

Wang, N. 2019. The citrus huanglongbing crisis and potential solutions. Mol. Plant 12:607-609. 